\title{
Enhanced cardiac inflammation and fibrosis in ovariectomized hypertensive rats: a possible mechanism of diastolic dysfunction in postmenopausal women
}

\author{
Takahiro Mori ${ }^{1}$, Hisashi Kai ${ }^{1}$, Hidemi Kajimoto ${ }^{2}$, Mitsuhisa Koga ${ }^{2}$, Hiroshi Kudo ${ }^{1}$, Narimasa Takayama $^{1}$, \\ Suguru Yasuoka ${ }^{1}$, Takahiro Anegawa ${ }^{1},{\text { Mamiko } \mathrm{Kai}^{3} \text { and Tsutomu Imaizumi }}^{1}$
}

Diastolic dysfunction is more prevalent in individuals with hypertension, particularly postmenopausal women; however, the pathogenesis of diastolic dysfunction remains unknown. Pressure overload activates cardiac inflammation, which induces myocardial fibrosis and diastolic dysfunction in rats with a suprarenal aortic constriction (AC). Therefore, we examined the effects of bilateral ovariectomy (OVX) on left ventricle (LV) remodeling, diastolic dysfunction and cardiac inflammation in hypertensive female rats. Rats were randomized to $\mathrm{OVX}+\mathrm{AC}$, OVX and $\mathrm{AC}$ groups as well as a Control group receiving sham operations for both the procedures. Rats underwent OVX at 6 weeks and AC at 10 weeks (Day 0). At Day 28, OVX did not appear to affect arterial pressure, cardiac hypertrophy or LV fractional shortening in AC rats. However, OVX increased myocardial fibrosis, elevated LV end-diastolic pressure and reduced the transmitral Doppler spectra early to late filling velocity ratio in AC rats. AC-induced transient myocardial monocyte chemoattractant protein-1 expression and macrophage infiltration, both of which peaked at Day 3 and were augmented and prolonged by OVX. At Day 28, dihydroethidium staining revealed superoxide generation in the intramyocardial arterioles in the OVX+AC group but not in the AC group. NOX1, a functional subunit of nicotinamide adenine dinucleotide phosphate oxidase, was upregulated only in the OVX+AC group at Day 28 . Chronic $17 \beta$-estradiol replacement prevented the increases in macrophage infiltration, NOX1 upregulation, myocardial fibrosis and diastolic dysfunction in $\mathrm{OVX}+\mathrm{AC}$ rats. In conclusion, we suggest that estrogen deficiency augments cardiac inflammation and oxidative stress and thereby aggravates myocardial fibrosis and diastolic dysfunction in hypertensive female rats. The findings provide insight into the mechanism underlying diastolic dysfunction in hypertensive postmenopausal women. Hypertension Research (2011) 34, 496-502; doi:10.1038/hr.2010.261; published online 20 January 2011

Keywords: estradiol; gender medicine; macrophage; myocardial fibrosis; oxidative stress

\section{INTRODUCTION}

The ejection fraction of the left ventricle (LV) is normal in approximately half of all patients with congestive heart failure, which indicates that impaired diastolic function in these patients is the major cause of heart failure. ${ }^{1-3}$ Hypertension is the most common coexisting disease in patients with diastolic heart failure. Diastolic heart failure is more prevalent in women than in men, especially in postmenopausal hypertensive women, which may be due to decreased estrogen levels. ${ }^{2,3}$

Determinants of diastolic function include active myocardial relaxation and passive properties of the LV wall. ${ }^{2}$ Sequestration of calcium and cross-bridge uncoupling after systole are involved in the active process of relaxation. Previous studies have shown that estrogen modulates calcium influx at L-type calcium channels, calcium uptake by the sarcoplasmic reticulum and myofibrillar calcium sensitivity, suggesting that estrogen deficiency may impair myocardial calcium handling and active LV relaxation. ${ }^{4}$ In addition to impaired active relaxation, there is emerging evidence that high passive LV stiffness has a role in diastolic dysfunction in postmenopausal women. ${ }^{5}$ Ventricular stiffness largely depends on excessive myocardial fibrosis; however, the effects of menopause on hypertensive myocardial fibrosis remain unclear. $^{6}$

In a previous study, we showed that Wistar rats with a suprarenal aortic constriction (AC) are an effective model for hypertensive cardiac hypertrophy associated with impaired diastolic but preserved

${ }^{1}$ Department of Internal Medicine, Division of Cardio-Vascular Medicine, Kurume University School of Medicine, Kurume, Japan; ${ }^{2}$ Cardiovascular Research Institute, Kurume University School of Medicine, Kurume, Japan and ${ }^{3}$ Department of Pharmaceutical Care and Health Sciences, Faculty of Pharmaceutical Sciences, Fukuoka University, Fukuoka, Japan

Correspondence: Dr H Kai, Department of Internal Medicine, Division of Cardio-Vascular Medicine, Kurume University School of Medicine, 67 Asahimachi, Kurume 830-0011, Japan.

E-mail: naikai@med.kurume-u.ac.jp

Received 24 September 2010; accepted 26 October 2010; published online 20 January 2011 
systolic function (see refs. 7, 8 for the review). In AC rats, pressure overload induces vascular superoxide generation and the induction of monocyte chemoattractant protein-1 (MCP-1), followed by perivascular macrophage infiltration. ${ }^{9,10}$ The inflammatory changes trigger reactive myocardial fibrosis, which is the major cause of diastolic dysfunction in this model. ${ }^{9,11,12}$ Estrogen deficiency augments MCP-1 expression and inflammation after vascular injury in rats undergoing bilateral ovariectomy (OVX). ${ }^{13}$ Thus, cardiac inflammatory changes may be enhanced by estrogen deficiency in individuals with hypertension, which may negatively affect diastolic dysfunction in postmenopausal females with hypertension. We investigated the effects of OVX on cardiac inflammation and LV remodeling and function in female $\mathrm{AC}$ rats.

\section{METHODS}

The study protocol was reviewed and approved by the Animal Care and Treatment Committee of Kurume University. The female Wistar rats were purchased from SLC (Shizuoka, Japan), housed under normal humidity, room temperature and a 12:12-h dark-light cycle and provided with unrestricted access to food and tap water.

\section{Study 1}

Animal model. This study included four groups: the OVX+AC group, which underwent OVX and AC; the OVX group, which underwent OVX and sham $\mathrm{AC}$; the AC group, which underwent $\mathrm{AC}$ and sham OVX; and the controls, which underwent sham operations representing both procedures. At 6 weeks of age, rats underwent bilateral OVX or a sham operation under intraperitoneal pentobarbital anesthesia $\left(50 \mathrm{mg} \mathrm{kg}^{-1}\right) .{ }^{14}$ At 10 weeks of age (Day 0$)$, AC was established with a ligature at the suprarenal abdominal aorta under $50 \mathrm{mg} \mathrm{kg}^{-1}$ intraperitoneal pentobarbital anesthesia. ${ }^{15,16}$

Blood pressure measurement and tissue preparation. Arterial pressure was measured in an unrestricted, conscious state using a heparinized polyethylene catheter that had been indwelled into the left carotid artery, and mean arterial pressure was calculated. ${ }^{11}$ Rats were euthanized with an overdose of intraperitoneal pentobarbital. For histological and immunohistological studies, rats ( $n=7$ per group per time point) were perfusion fixed with $4 \%$ glutaraldehyde in Hank's solution at $100 \mathrm{~mm} \mathrm{Hg}$, and the LV was immediately excised and embedded in paraffin. ${ }^{11}$ For dihydroethidium staining and real-time reversetranscription (RT)-PCR, rats ( $n=12$ per group per time point) were perfused with ice-cold saline at $100 \mathrm{~mm} \mathrm{Hg}$ for $5 \mathrm{~min}$, and the LV was immediately excised, snap frozen in liquid nitrogen, and stored at $-80{ }^{\circ} \mathrm{C}$ until use

\section{Morphometry and immunohistostaining}

Paraffinized sections were processed for morphometry and immunohistostaining. In three independent hematoxylin-eosin-stained sections, the shortest transverse myocyte diameter was measured in 50 nucleated transverse sections of the myocytes in each rat. ${ }^{17}$ The percent area of myocardial fibrosis was evaluated in three independent Mallory-Azan-stained sections of each rat. ${ }^{18}$ Immunohistostaining for monocytes/macrophages was performed using an antibody against ED1 (Chemicon International, Temecula, CA, USA) and a commercially available detection system (DAKO, Glostrup, Denmark). ${ }^{9,19}$ The number of macrophages were counted at $\times 200$ magnification in four independent, whole cross-sections in each rat.

Dihydroethidium staining. Fresh frozen LV sections ( $n=5$ per group) were stained with dihydroethidium (Invitrogen, Carlsbad, CA, USA), a fluorescent dye assessing superoxide formation, as previously described..$^{10}$ Imaging was performed using an epifluorescence microscope (Olympus, Tokyo, Japan).

Real-time RT-PCR. Quantitative analysis of mRNA expressions was performed using real-time RT-PCR. ${ }^{11}$ Total RNA was extracted from the frozen hearts ( $n=7$ per group per time point) using TRIzol (Invitrogen).$^{20,21}$ Aliquots of total RNA were reverse transcribed and amplified in triplicate using the SYBER PrimeScript RT-PCR kit (TAKARA BIO, Kyoto, Japan) and a Sequence Detection System Model 7700 (Applied Biosystems, Foster city, CA, USA).
Primer pairs and probes for MCP-1, NOX1, endothelial nitric oxide synthase (eNOS), transforming growth factor- $\beta 1$ (TGF- $\beta 1$ ), activin receptor-like kinase5 (TGF- $\beta$ type I receptor), TGF- $\beta$ type II receptor and $\beta$ actin were purchased from TAKARA BIO. The expression level of the target gene was normalized to the $\beta$-actin level in each sample.

Echocardiographic studies. At Day 28, rats ( $n=7$ per group) were anesthetized with intraperitoneal ketamine $\left(50 \mathrm{mg} \mathrm{kg}^{-1}\right)$ and xylazine $\left(10 \mathrm{mg} \mathrm{kg}^{-1}\right)$. LV fractional shortening and the early to late filling velocity ratio $(\mathrm{E} / \mathrm{A})$ were measured at Day 28 using an echocardiograph equipped with a $10-\mathrm{MHz}$ transducer (Aloka, Tokyo, Japan). ${ }^{11,22,23}$

Hemodynamic measurement. At Day 28 , rats ( $n=7$ per group) were intraperitoneally anesthetized with $15 \mathrm{mg} \mathrm{kg}^{-1}$ pentobarbital. A 2-F micromanometertipped catheter (Millar Instruments, Burnaby, BC, Canada) was inserted retrogradely into the $\mathrm{LV}$ through the left carotid artery, and the left ventricle end-diastolic pressure (LVEDP) was measured during spontaneous breathing. ${ }^{11}$

\section{Study 2}

The female Wistar rats were randomized to AC, OVX+AC and OVX+AC+estradiol (E2) groups ( $n=21$ per group).

$17 \beta$-Estradiol replacement experiment. OVX and AC were performed as described above. $\mathrm{OVX}+\mathrm{AC}+\mathrm{E} 2$ rats and $\mathrm{OVX}+\mathrm{AC}$ rats received daily subdermal injections of E2 $\left(20 \mu \mathrm{g} \mathrm{kg}^{-1}\right.$ per day) and the vehicle starting on the day after OVX. A previous study showed that this dose of E2 resulted in physiological levels of circulating E2 in rats after OVX. ${ }^{24}$ Echocardiographic and hemodynamic studies, histological analyses, immunohistostaining and real-time RT-PCR were performed as described above.

Statistical analysis. Each quantitative morphometry and immunohistostaining analysis was performed by three observers in a blind manner. Intra- or interobserver variability was $<5 \%$ in each experiment. We performed twofactor factorial analysis of variance followed by Scheffe's F-test for statistical comparisons. For the E2 replacement experiment, one-way analysis of variance followed by Scheffe's F-test was used. A value of $P<0.05$ was considered significant.

\section{RESULTS}

Study 1

Blood pressure and LV hypertrophy. AC resulted in an initially rapid increase followed by a more gradual increase in arterial pressure, which was associated with a progressive increase in the ratio of left ventricle weight to body weight ( $\mathrm{LVW} / \mathrm{BW}$ ), an indicator of $\mathrm{LV}$ hypertrophy (Figure 1A). OVX did not affect arterial pressure and LVW/BW. As BW was increased by OVX (data not shown), it is possible that the LVW/BW ratio may have underestimated LV hypertrophy in the OVX and OVX+AC groups. Thus, the ratio of LVW to tibial length (LVW/TL) was also evaluated (Figure 1Ac). The results evaluated by LVW/BW and LVW/TL were similar.

Echocardiographic and hemodynamic measurement. The echocardiography and cardiac catheterization were performed at Day 28 (Figure 1B). Neither OVX nor AC affected LV fractional shortening. The E/A ratio and LVEDP were similar in Control and OVX groups. $\mathrm{AC}$ resulted in a significantly decreased E/A ratio, which was further decreased by OVX. OVX itself did not affect LVEDP. AC resulted in elevated LVEDP, which was further elevated in the OVX+AC group.

\section{Myocardial remodeling}

Histological analysis was performed for myocardial remodeling at Day 28 (Figure 2). The myocyte diameter was similar in the Control and OVX groups (Figure 2Ba). AC resulted in myocyte hypertrophy, which was not affected by OVX. Myocardial fibrosis was sparse in Control and OVX groups (Figure 2A). AC resulted in reactive myocardial fibrosis, which expanded from the perivascular space to the 

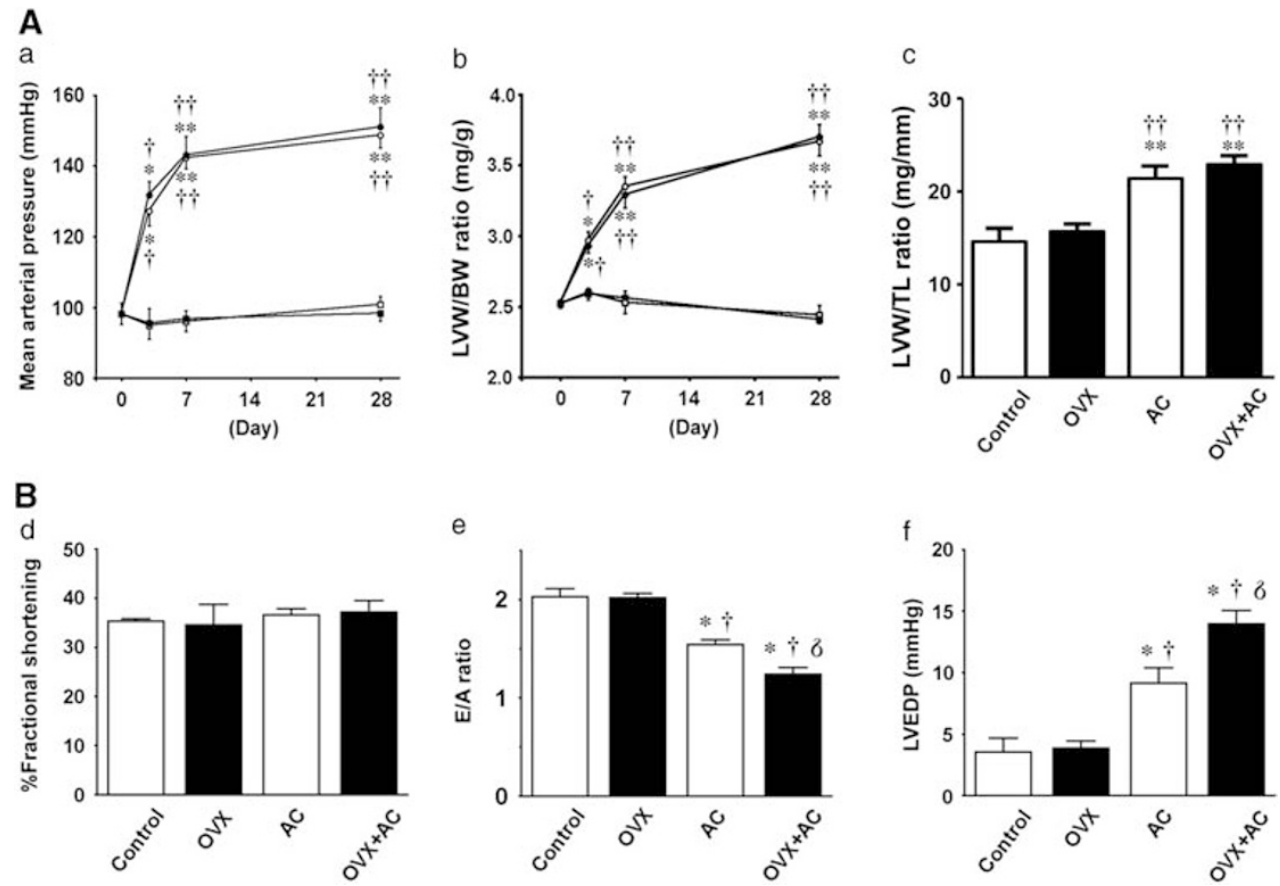

Figure 1 (A) Temporal changes in mean arterial pressure (a) and the ratio of left ventricle (LV) weight to body weight (LVW/BW) (b). Open square, Control group; closed square, ovariectomy $(\mathrm{OVX})$ group; open circle, aortic constriction $(\mathrm{AC})$ group and closed circle, OVX+AC group. The ratio of left ventricle weight to tibial length (LVW/TL) (c) at Day 28. (B) Effects of OVX and AC on fractional shortening (a), early to late filling velocity (E/A) ratio (b), and left ventricle end-diastolic pressure (LVEDP) (c). Bar=1 s.d. ( $n=7$ per group). ${ }^{*} P<0.05$ and ${ }^{* *} P<0.01$ vs. Control; ${ }^{\dagger} P<0.05$ and ${ }^{\dagger \dagger} P<0.01$ vs. OVX; ${ }^{\delta} P<0.05$ vs. AC.
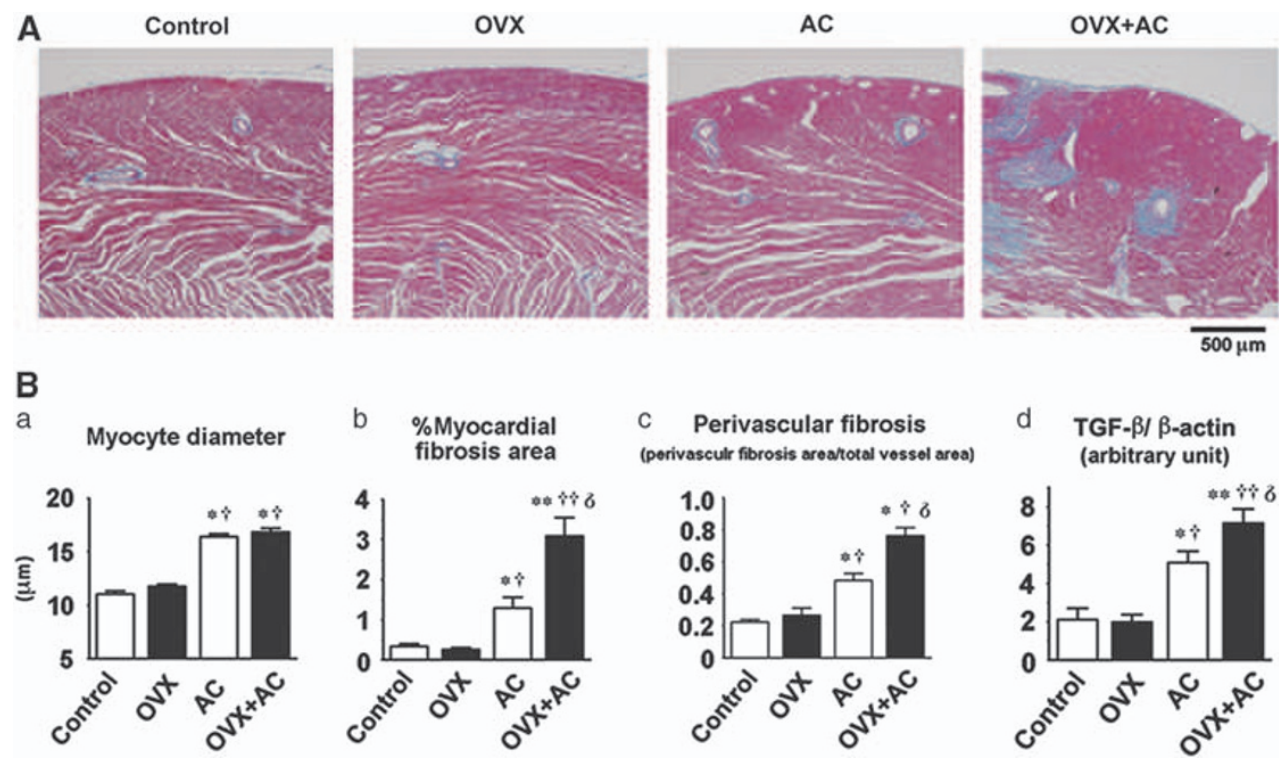

Figure 2 (A) Representative microphotographs of Mallory-Azan-stained cross-sections of the heart at Day 28. Fibrotic tissue was stained blue. (B) Pooled data showing the effects of ovariectomy (OVX) and aortic constriction (AC) on transverse myocyte diameter (a), percent myocardial fibrosis area (b), perivascular fibrosis (c), and transforming growth factor- $\beta 1$ (TGF- $\beta 1$ ) mRNA expression (d). The expression level of the target gene was normalized by the $\beta$ actin level in each sample. Bar=1 s.d. ( $n=7$ per group). ${ }^{*} P<0.05$ and ${ }^{* *} P<0.01$ vs. Control; ${ }^{\dagger} P<0.05$ and ${ }^{\dagger \dagger} P<0.01$ vs. 0 VX; ${ }^{\delta} P<0.05$ vs. $A$.

intermuscular interstitium and was associated with increases in myocardial and perivascular fibrosis (Figure 2B). OVX remarkably increased AC-induced myocardial fibrosis. Myocardial expression levels of TGF- $\beta 1$ were investigated at Day 7 (Figure 2Bd) because AC caused a transient TGF- $\beta 1$ induction in male rats with a peak at
Day $7 .{ }^{11}$ TGF- $\beta 1$ mRNA expression was similar in Control and OVX groups. AC upregulated TGF- $\beta 1$ expression, which was further upregulated by OVX. In contrast, AC and OVX did not affect the mRNA expressions of TGF- $\beta 1$ receptors, activin receptor-like kinase- 5 and TGF- $\beta$ type II receptor (data not shown). 


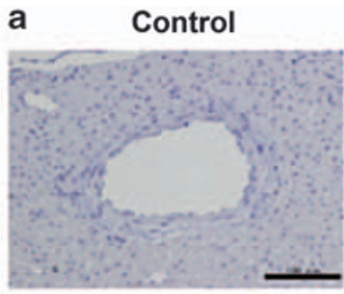

AC

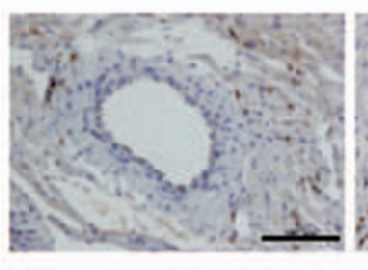

b
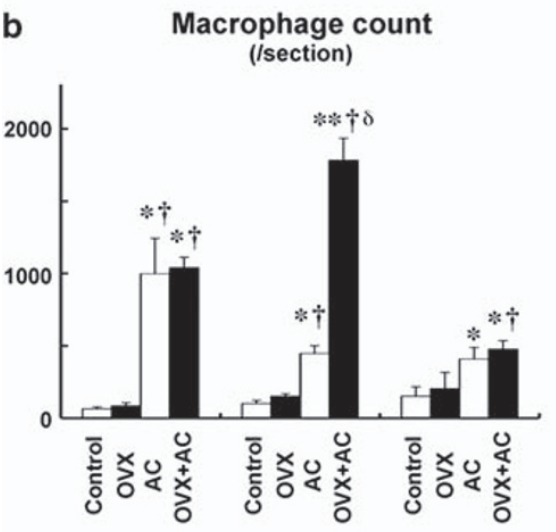

Day 3

Day 7

Day 28

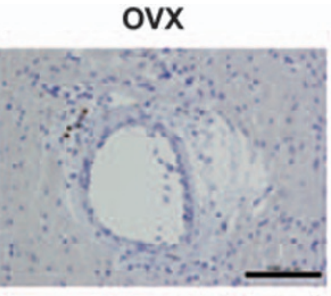

OVX+AC
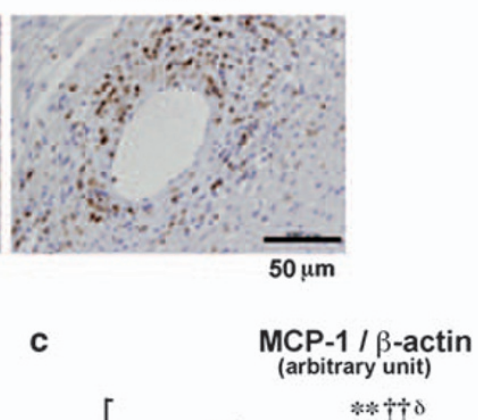

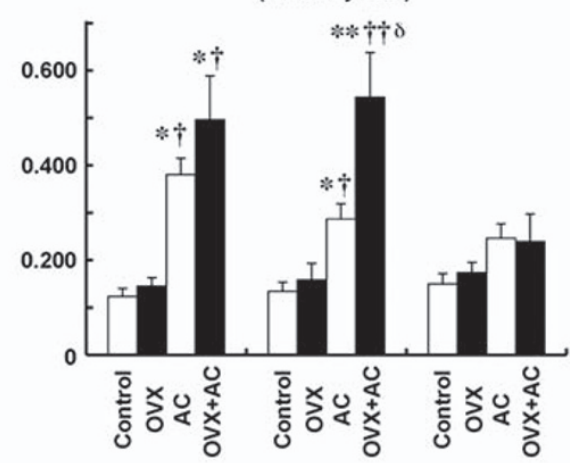

Day 3

Day 7

Day 28

Figure 3 (a) Representative microphotographs of immunohistostaining for ED1+ macrophages (stained as brown) at Day 7 . Temporal changes in the number of ED1+ macrophages (b) and monocyte chemoattractant protein-1 (MCP-1) mRNA expression (c). Bar=1 s.d. ( $n=7$ per group). ${ }^{*} P<0.05$ and ${ }^{* *} P<0.01$ vs. Control; ${ }^{\dagger} P<0.05$ and ${ }^{\dagger \dagger} P<0.01$ vs. ovariectomy (OVX); ${ }^{\delta} P<0.05$ vs. aortic constriction (AC).

Cardiac inflammatory changes. The ED1+ macrophages were scarce in Control and OVX groups (Figure 3a). Consistent with a previous study, ${ }^{9} \mathrm{AC}$ induced a transient macrophage infiltration in the perivascular space with a peak at Day 3 and a decrease at Day 28, which was still significantly higher than the Control group (Figure $3 \mathrm{~b}$ ). OVX augmented and prolonged AC-induced macrophage infiltration, which peaked at Days 3-7. The macrophage count declined to a level similar to AC rats at Day 28.

MCP-1 mRNA expression did not differ between Control and OVX groups (Figure 3c). AC transiently upregulated MCP-1 expression, which peaked at Day 3 and returned to the level of the Control group at Day 28. OVX enhanced AC-induced MCP-1 induction; peak expression was prolonged from Days 3 to 7 and declined to the level of the AC rats at Day 28.

\section{Superoxide generation and eNOS expression}

In the Control group, dihydroethidium signals were scarcely detected in the heart. ${ }^{10} \mathrm{AC}$ resulted in increased dihydroethidium signals in the intramyocardial arterial wall and, to a lesser extent, in the myocardium at Day 3 (Figure 4A). The AC-induced dihydroethidium signals disappeared at Day 28. Dihydroethidium signals were observed in the OVX+AC group at Days 3 and 28. OVX itself did not increase dihydroethidium signals during the observation period (data not shown). At Day 28, mRNA expression of NOX1, the functional subunit of nicotinamide adenine dinucleotide phosphate oxidase of vascular smooth muscle cells, ${ }^{25}$ was remarkably upregulated in the OVX+AC group but did not differ among the Control, OVX and AC groups (Figure $4 \mathrm{Ba}$ ). We also examined eNOS mRNA expression at Day 28 (Figure $4 \mathrm{Bb}$ ). The Control and AC groups demonstrated similar eNOS expression levels. OVX reduced the eNOS expression level, which was not affected by AC.

\section{Study 2}

The effects of chronic E2 administration were examined in OVX+AC rats to investigate whether the OVX-induced changes were attributable to estrogen deficiency. Preliminary experiments showed that E2 replacement did not change mean arterial pressure, LVW/BW and myocardial fibrosis in AC rats not receiving OVX (data not shown).

Effects of $17 \beta$-estradiol replacement in $O V X+A C$ rats. At Day 28, there were no differences in arterial pressure, LVW/BW, LVW/TL and myocyte diameter among the $\mathrm{AC}, \mathrm{OVX}+\mathrm{AC}$ and $\mathrm{OVX}+\mathrm{AC}+\mathrm{E} 2$ groups (Figures 5A and 5Ba). E2 replacement prevented OVX-induced myocardial fibrosis in $\mathrm{AC}$ rats (Figures $5 \mathrm{Bb}$ and $5 \mathrm{Bc}$ ). Moreover, the OVX-induced increase in macrophage infiltration was counteracted by E2 replacement at Day 7 (Figure 6A). E2 replacement also prevented 

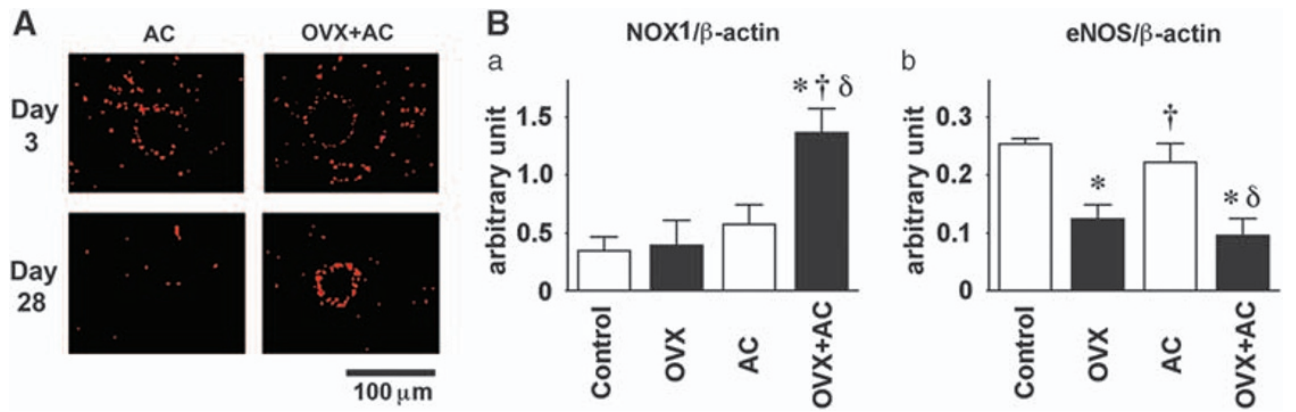

Figure 4 (A) Representative microphotographs of dihydroethidium staining at Days 3 and 28. Red signals represent superoxide generation. (B) mRNA expression levels of NOX1 (a) and endothelial nitric oxide synthase (eNOS) (b) at Day 28. Bar=1 s.d. $(n=7) .{ }^{*} P<0.05$ and ${ }^{* *} P<0.01$ vs. control; ${ }^{\dagger} P<0.05$ vs. ovariectomy (OVX); ${ }^{\delta} P<0.05$ vs. aortic constriction (AC).
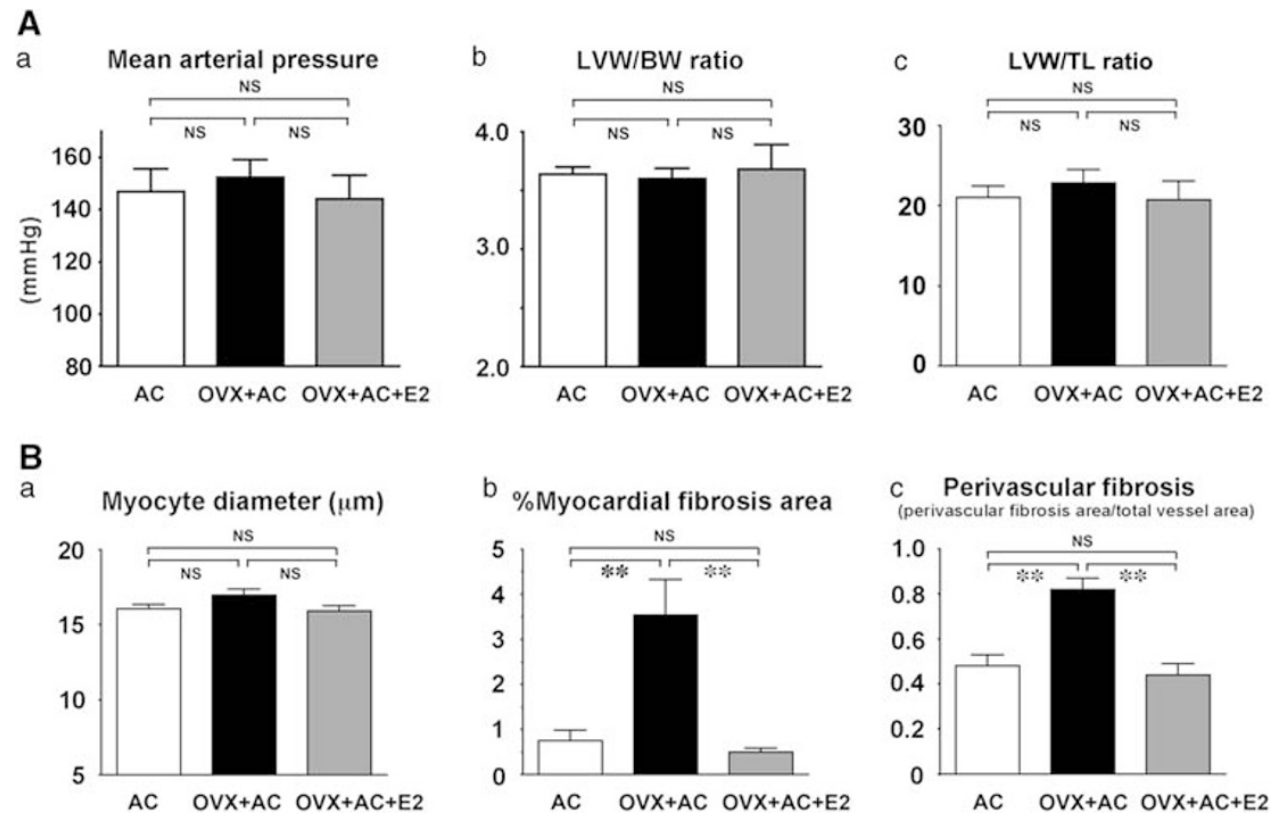

Figure 5 (A) Effects of $17 \beta$-estradiol (E2) replacement on mean arterial pressure (a), left ventricle weight to body weight (LVW/BW) (b), and left ventricle weight to body weight (LVW/TL) (c) at Day 28. (B) Effects of E2 replacement on myocyte diameter (a), percent myocardial fibrosis area (b), and perivascular fibrosis (c) at Day 28. (c) Bar=1 s.d. ( $n=7$ per group). ${ }^{* *} P<0.01$. NS, not statistically significant.

AC-induced NOX1 upregulation and eNOS downregulation in OVX rats at Day 28 (Figure 6B). E2 replacement prevented the OVXinduced E/A reduction and LVEDP elevation without affecting fractional shortening at Day 28 (Figure 6C).

\section{DISCUSSION}

This study showed for the first time that OVX augments pressure overload-induced cardiac inflammation and superoxide generation. Moreover, OVX aggravated myocardial fibrosis, but not the myocyte hypertrophy, which resulted in increased diastolic dysfunction in AC rats. The effects of OVX were offset by chronic E2 replacement, indicating that estrogen deficiency was the major cause of the observed changes in OVX $+\mathrm{AC}$ rats.

Our previous studies have shown that the male AC rat is an effective model for hypertensive LV hypertrophy exhibiting preserved systolic and impaired diastolic function., ${ }^{7,8}$ The histological features of this model are myocyte hypertrophy and reactive myocardial fibrosis, which progressively expands from the perivascular space to the interstitial tissue surrounding the muscle fiber bundles. ${ }^{11}$ These histological changes mimic myocardial remodeling observed in patients at early stages of hypertensive heart disease. ${ }^{26}$ Using this model, we demonstrated that a series of inflammatory changes are induced by pressure overload around the intramyocardial arteries. Acute increases in pressure occur concurrently with transient inductions of MCP-1 and superoxide generation in the intramyocardial arteries associated with perivascular macrophage infiltration (Days 1-3). MCP-1 expression, superoxide generation and the number of accumulated macrophages gradually decrease while arterial pressure remains elevated. ${ }^{9,10}$ AC-induced inflammatory changes result in myocardial fibrosis in the early phase and diastolic dysfunction in the later phase. ${ }^{9,11,12,19}$ In this study, AC induced cardiac remodeling and diastolic dysfunction associated with inflammatory changes and superoxide generation in control female rats. A growing body of evidence implicates vascular inflammation in cardiovascular diseases in postmenopausal hypertensive women. ${ }^{13,27}$ Thus, it is possible that estrogen deficiency in postmenopausal women may augment cardiac 
A

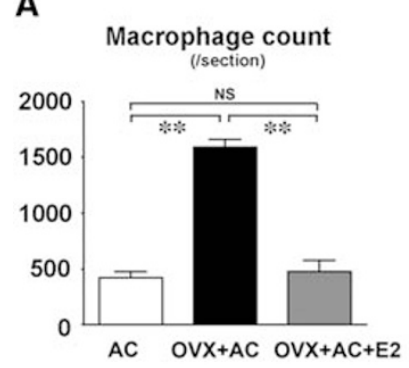

C

a \%Fractional shortening

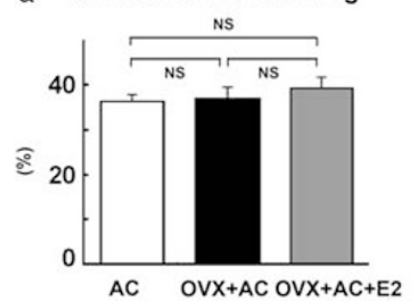

B

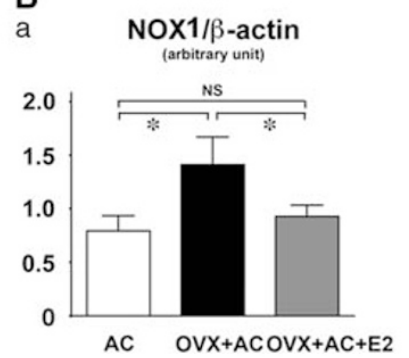

b

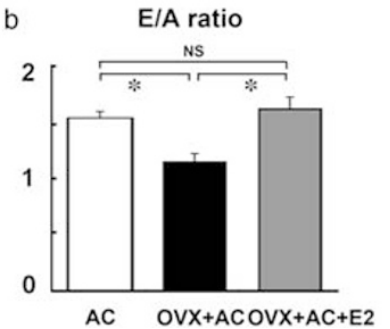

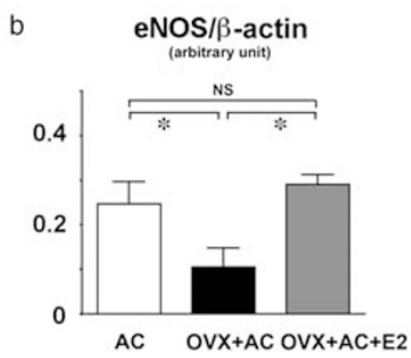

C

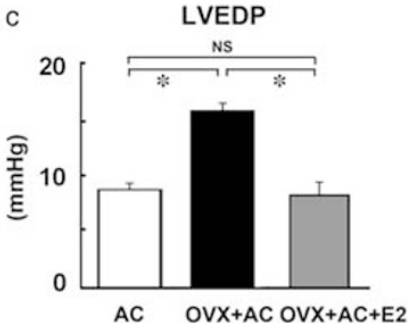

Figure 6 (A) Effects of E2 replacement on the count of ED1+ macrophages at Day 7. (B) Effects of E2 replacement on mRNA expression of NOX1 (A) and eNOS (B) at Day 28. (C) Effects of E2 replacement on fractional shortening (a), early to late filling velocity (E/A) ratio (b), and left ventricle end-diastolic pressure (LVEDP) (c) at Day 28 . Bar $=1$ s.d. ( $n=7$ per group). ${ }^{*} P<0.05$ and ${ }^{*} P<0.01$. NS, not statistically significant.

inflammation and aggravate myocardial fibrosis, which may lead to LV diastolic dysfunction. We investigated the effects of OVX on cardiac inflammation and LV remodeling and function in female AC rats.

As shown in Figure 1, OVX aggravated diastolic dysfunction without significantly changing systolic function in the pressure-overloaded heart of female rats. Thus, OVX+AC rats may be used as a model of postmenopausal hypertension. OVX markedly increased AC-induced myocardial fibrosis and upregulation of TGF- $\beta 1$ (Figure 2). We have previously shown that the diastolic dysfunction is attributed mainly to TGF- $\beta$-mediated reactive myocardial fibrosis in AC rats. ${ }^{11}$ In this study, arterial pressure and LV/myocyte hypertrophy were not changed by OVX (Figures 1 and 2). Thus, estrogen deficiency may induce further diastolic dysfunction in AC rats by aggravating myocardial fibrosis. It has also been shown that oxidative stress contributes to the proliferation, phenotypic transition and collagen synthesis of cardiac fibroblasts. ${ }^{28}$ Thus, OVX-augmented oxidative stress might aggravate TGF- $\beta$-mediated myocardial fibrosis in pressure-overloaded hearts.

The novel finding of this study was that the estrogen deficiency enhanced pressure overload-induced cardiac inflammation. We have shown that pressure overload induces MCP-1 induction and macrophage infiltration, which peaked at Day 3 in male AC rats. ${ }^{9}$ Similar results were found in female AC rats without OVX (Figure 4). Interestingly, estrogen deficiency not only increased MCP-1 induction and macrophage infiltration but also prolonged the duration of these changes. These observations are consistent with a previous report that estrogen deficiency augmented MCP-1-mediated vascular inflammation, which aggravates neointima formation in a rat balloon-injured artery. ${ }^{13}$ A causal relationship between inflammation and reactive myocardial fibrosis and diastolic dysfunction has been established in AC rats: a neutralizing antibody against MCP-1 or intercellular adhesion molecule-1 not only abolished macrophage infiltration but also inhibited fibroblast proliferation, TGF- $\beta$ upregulation and myocardial fibrosis in AC rats. ${ }^{9,12}$ Anti-MCP-1 neutralizing antibody prevented the progression of diastolic dysfunction in $\mathrm{AC}$ rats. ${ }^{9}$ The augmentation of cardiac inflammation induced by estrogen deficiency is implicated in the aggravation of myocardial fibrosis and diastolic dysfunction in OVX+AC rats. In addition to the enhancement of inflammation, the direct effects of estrogen deficiency may be responsible for increased myocardial fibrosis in AC rats because E2 inhibits collagen expression in cultured cardiac fibroblasts. ${ }^{29}$

The mechanism by which estrogen deficiency enhances pressure overload-induced cardiac inflammation was not determined in this study. It has been shown that OVX enhances vascular inflammation by inducing superoxide generation in balloon-injured but not intact arteries, which indicates that oxidative stress may have a role in the augmentation of vascular inflammation by estrogen deficiency. ${ }^{13}$ In AC rats, vascular superoxide generation and macrophage infiltration occur simultaneously, followed by reactive myocardial fibrosis. ${ }^{10}$ Sustained superoxide generation associated with NOX1 induction was observed in OVX+AC rats, although superoxide generation was transient in the early phase in female AC rats without OVX (Figure 5). It has been shown that reactive oxygen species mediate cell-to-cell interactions between macrophages and fibroblasts, which, in turn, augment fibro-inflammatory changes. ${ }^{30}$ Thus, sustained oxidative stress via vascular nicotinamide adenine dinucleotide phosphate oxidase activation may exaggerate perivascular inflammation and reactive myocardial fibrosis in $\mathrm{OVX}+\mathrm{AC}$ rats.

Postmenopausal females may become more susceptible to cardiovascular diseases through reduced eNOS expression and activity. ${ }^{4}$ OVX resulted in decreased eNOS expression (Figure $5 \mathrm{Bb}$ ) and augmented vascular inflammation in balloon-injured rat arteries. ${ }^{13}$ Thus, eNOS reduction may result in the augmentation of inflammatory changes in OVX+AC rats. Endogenous estrogens are powerful antioxidants, which inhibit the generation of reactive oxygen species and increase the bioavailability of nitric oxide. ${ }^{31}$ Thus, it is possible that eNOS reduction by estrogen deficiency may augment AC-induced inflammation not only directly but also indirectly by chronically increasing superoxide generation. A combination of pressure overload and eNOS reduction may have activated unknown mechanisms to augment inflammation and myocardial fibrosis in OVX+AC rats. It is 
possible that the aggravation of the local angiotensin II system by estrogen deficiency may affect hypertensive cardiac remodeling in our model. These issues should be addressed in a future study.

\section{Limitation of this study}

It has been shown that aging reduces the cardiovascular protective and anti-inflammatory effects of estrogen. ${ }^{27}$ Therefore, it is possible that the beneficial effects of estrogen replacement in this study might have been limited in older rats.

In conclusion, estrogen deficiency not only augmented cardiac inflammation and oxidative stress but also aggravated myocardial fibrosis and diastolic dysfunction in hypertensive female rats. These findings raise the possibility that cardiac inflammation may be a target for preventing diastolic dysfunction in females with hypertension. Our current results do not necessarily warrant the efficacy of estrogen replacement therapy in the treatment of diastolic dysfunction in the postmenopausal hypertensives.

\section{CONFLICT OF INTEREST}

The authors declare no conflict of interest.

\section{ACKNOWLEDGEMENTS}

We thank Kaoru Moriyama, Reiko Fujiyoshi, Michiko Taguchi, Miho Kogure, Miyuki Nishigata, Kimiko Kimura, Makiko Kiyohiro and Katsue Shiramizu for their skillful technical assistance. This study was supported in part by the Strategic Support Program for Research Infrastructure Development of Private Universities, the Science Frontier Research Promotion Centers Program, and Grants-In-Aid for Scientific Research from the Ministry of Education, Science, Sports and Culture, Japan. This work was also partially supported by grants from Sharyo-Kyogi Foundation, Kimura Memorial Heart Foundation, Ishibashi Memorial Foundation, Takeda Science Foundation and Kakihara Science Foundation.

1 Vasan RS, Benjamin EJ, Levy D. Prevalence, clinical features and prognosis of diastolic heart failure: an epidemiologic perspective. J Am Coll Cardiol 1995; 26: 1565-1574.

2 Ouzounian M, Lee DS, Liu P. Diastolic heart failure: mechanisms and controversies. Nat Clin Pract Cardiovasc Med 2008; 5: 375-386.

3 Maeder MT, Kaye DM. Heart failure with normal left ventricular ejection fraction. J Am Coll Cardiol 2009; 53: 905-918.

4 Regitz-Zagrosek V, Brokat S, Tschope C. Role of gender in heart failure with normal left ventricular ejection function. Progress Cardiovasc Dis 2007; 49: 241-251.

5 Hayward CS, Kalnins WV, KeLLy RP. Gender-related difference in left ventricular chamber function. Cardiovasc Res 2001; 49: 340-350.

6 Covell JW. Factors influencing diastolic function. Possible role of the extracellular matrix. Circulation 1990; 81: III155-III158.

7 Kai H, Kuwahara F, Tokuda K, Imaizumi T. Diastolic dysfunction in hypertensive hearts: roles of perivascular inflammation and reactive myocardial fibrosis. Hypertens Res 2005; 28: 483-490.

8 Kai H, Kuwahara F, Tokuda K, Imaizumi T. Perivascular inflammation and hypertensive cardiovascular remodeling. Curr Hypertens Rev 2006; 2: 263-269.

9 Kuwahara F, Kai H, Tokuda K, Takeya M, Takeshita A, Egashira K, Imaizumi T. Hypertensive myocardial fibrosis and diastolic dysfunction: another model of inflammation. Hypertension 2004; 43: 739-745.

10 Kai H, Mori T, Tokuda K, Takayama N, Tahara N, Takemiya K, Kudo H, Sugi Y, Fukui D, Yasukawa H, Kuwahara F, Imaizumi T. Pressure overload-induced transient oxidative stress mediates perivascular inflammation and cardiac fibrosis through angiotensin II. Hypertens Res 2006; 29: 711-718.

11 Kuwahara F, Kai H, Tokuda K, Kai M, Takeshita A, Egashira K, Imaizumi T. Transforming growth factor- $\beta$ function blocking prevents myocardial fibrosis and diastolic dysfunction in pressure-overloaded rats. Circulation 2002; 106: 130-135.

12 Kuwahara F, Kai H, Tokuda K, Niiyama H, Tahara N, Kusaba K, Takemiya K, Jalalidin A, Koga M, Nagata T, Shibata R, Imaizumi T. Roles of intercellular adhesion molecule-1 in hypertensive cardiac remodeling. Hypertension 2003; 41: 819-823.

13 Miller AP, Feng W, Xing D, Weathington NM, Blalock JE, Chen YF, Oparil S. Estrogen modulates inflammatory mediator expression and neutrophil chemotaxis in injured arteries. Circulation 2004; 110: 1664-1669.

14 Kai M, Tominaga K, Okimoto K, Yamauchi A, Kai H, Kataoka Y. Ovariectomy aggravates nifedipine-induced flushing of tail skin in mice. Eur J Pharmacol 2003; 481: 79-82.

15 Limas CJ. Increased number of $\beta$-adrenergic receptors in the hypertrophied myocardium. Biochem Biophys Acta 1979; 588: 174-178.

16 Kuwahara F, Kai H, Tokuda K, Shibata R, Kusaba K, Tahara N, Niiyama H, Nagata T, Imaizumi T. Hypoxia-inducible factor-1 $\alpha /$ vascular endothelial growth factor pathway for adventitial vasa vasorum formation in hypertensive rat aorta. Hypertension 2002; 39: 46-50.

17 Kai H, Muraishi A, Sugiu Y, Nishi H, Seki Y, Kuwahara F, Kimura A, Kato H, Imaizumi T. Expression of proto-oncogenes and gene mutation of sarcomeric proteins in patients with hypertrophic cardiomyopathy. Circ Res 1998; 83: 594-601.

18 Takemoto M, Egashira K, Tomita H, Usui M, Okamoto H, Kitabatake A, Shimokawa H, Sueishi K, Takeshita A. Chronic angiotensin-converting enzyme inhibition and angiotensin II type 1 receptor blockade. Effects on cardiovascular remodeling in rats induced by the long-term blockade of nitric oxide synthesis. Hypertension 1997; 30: 1621-1627.

19 Tokuda K, Kai H, Kuwahara F, Yasukawa H, Tahara N, Kudo H, Takemiya K, Koga M, Yamamoto T, Imaizumi T. Pressure-independent effects of angiotensin II on hypertensive myocardial fibrosis. Hypertension 2004; 43: 499-503.

20 Kusaba K, Kai H, Koga M, Takayama N, Ikeda A, Yasukawa H, Seki Y, Egashira K, Imaizumi T. Inhibition of intrinsic interferon- $\gamma$ function prevents neointima formation after balloon injury. Hypertension 2007; 49: 909-915.

21 Koga M, Kai H, Yasukawa H, Yamamoto T, Kawai Y, Kato S, Kusaba K, Kai M, Egashira $\mathrm{K}$, Kataoka $\mathrm{Y}$, Imaizumi T. Inhibition of progression and stabilization of plaques by therapeutic interferon- $\gamma$ function blocking in ApoE-KO mice. Circ Res 2007; 101: 348-356.

22 Kudo H, Kai H, Kajimoto H, Koga M, Takayama N, Mori T, Ikeda A, Yasuoka S, Anegawa T, Mifune H, Kato S, Hirooka H, Imaizumi T. Exaggerated blood pressure variability superimposed on hypertension aggravates cardiac remodeling in rats via angiotensin II system-mediated chronic inflammation. Hypertension 2009; 54: 832-838.

23 Takayama N, Kai H, Kudo H, Yasuoka S, Mori T, Anegawa T, Koga M, Kajimoto H, Hirooka Y, Imaizumi T. Simvastatin prevents large blood pressure variability induced aggravation of cardiac hypertrophy in hypertensive rats by inhibiting RhoA/Ras-ERK pathways. Hypertens Res 2011; 34: 71-77.

24 Chen SJ, Li H, Durand J, Oparil S, Chen YF. Estrogen reduces myointimal proliferation after balloon injury of rat carotid artery. Circulation 1996; 93: 577-584.

25 Lassegue B, Sorescu D, Szocs K, Yin Q, Akers M, Zhang Y, Grant SL, Lambeth JD, Griendling KK. Novel gp91(phox) homologues in vascular smooth muscle cells: Nox1 mediates angiotensin II-induced superoxide formation and redox-sensitive signaling pathways. Circ Res 2001; 88: 888-894.

26 Weber KT, Brilla CG, Janicki JS. Myocardial fibrosis: functional significance and regulatory factors. Cardiovasc Res 1993; 27: 341-348.

27 Xing D, Nozell S, Chen Y-F, Hage F, Oparil S. Estrogen and mechanisms of vascular protection. Arteriol Thromb Vasc Biol 2009; 29: 289-295.

28 Siwik DA, Pagano PJ, Colucci WS. Oxidative stress regulates collagen synthesis and matrix metalloproteinase activity in cardiac fibroblasts. Am J Physiol Cell Physiol 2001; 280: C53C60.

29 Dubey RK, Gillespie DG, Jackson EK, Keller PJ. 17Beta-estradiol, its metabolites, and progesterone inhibit cardiac fibroblast growth. Hypertension 1998; 31(1 Part 2): 522-528.

30 Steinhauser ML, Kunkel SL, Hogaboam CM, Evanoff H, Strieter RM, Lukacs NW. Macrophage/fibroblast coculture induces macrophage inflammatory protein-1alpha production mediated by intercellular adhesion molecule- 1 and oxygen radicals. J Leukoc Biol 1998; 64: 636-641.

31 Meyer MR, Haas E, Barton M. Gender differences of cardiovascular diseases: new perspectives for estrogen receptor signaling. Hypertension 2006; 47: $1019-1026$. 